\title{
REAL HUMAN FACE DETECTION FOR SURVEILLANCE SYSTEM USING HETEROGENEOUS SENSORS
}

\author{
Yoon-Ki Kim ${ }^{1}$, Doo-Hyun Hwang ${ }^{2}$ and Chang-Sung Jeong ${ }^{3}$ \\ ${ }^{1,2,3}$ Department of Electrical Engineering, Korea University, Seoul, South Korea \\ ${ }^{1}$ vardinekorea.ac.kr \\ ${ }^{2}$ doohh88akorea.ac.kr \\ ${ }^{3} \mathrm{csjeong}$ jkorea.ac.kr
}

\begin{abstract}
Face detection algorithms are used to detect the human in various industry fields. A typical face detection algorithm such as Haar Feature-based Cascade Classifier gives us an easier way to detect human face. It consists of several classifiers which contain complicated arithmetic operations. Several classifiers constitute the cascade which can detect each element of human face. The more cascades are contained in the algorithm to detect elements of human face, the more it takes a time to detect human face. The previous cascade hardly recognize real human, since previous cascade processes only one source from image source. In this paper, we present a new cascade method for human face detection which exploits several classifiers for data not only from image source but also various heterogeneous sensors. Cascades consist of various sensors based on tuple data type could be operated quickly. It provides more accuracy of real human face detection, reduces the number of classifier for high speed processing in real-time.
\end{abstract}

\section{KEYWORDS}

Face Detection, Heterogeneous Sensor, Real-Time Processing, Haar-Like Feature

\section{INTRODUCTION}

In Internet of Things environment with wired/wireless sensor networks, efficient sensor data process are very significant for various useful data analysis [1]. Various sensors such as CCTVs, thermo-graphic camera and temperature sensors can be processed at the same time for more accuracy analysis. Those sensors notice different signal respectively. For example, CCTV notices image signal to detect face shape, thermo-graphic camera notices image signal to detect face temperature and gas sensor notices amount of gas in air. This heterogeneous sensors detect not only one sense but also various senses. Various sensors can enhance the accuracy of real human face detection in real-time environment.

Haar Feature-base Cascade is a useful algorithm in wide range of object detection application [2]. Cascading is a particular case of ensemble learning based on the concatenation of several classifiers, using all information collected from the output from a given classifier as additional information for the next classifier in the cascade [3]. In face detection field, classifier processes David C. Wyld et al. (Eds) : NETCOM, NCS, WiMoNe, CSEIT, SPM - 2015

pp. 203-210, 2015. @ CS \& IT-CSCP 2015

DOI : $10.5121 / \mathrm{csit} .2015 .51617$ 
multimedia data from one source. A typical method is that first classifier detects the face shape, and then next classifiers can detect other shape such as eyes, mouth and nose in face shape. However, the more cascades are contained in that, the more it takes a time to finish. Consequentially, there is a trade-off between accuracy of result and processing speed. In this paper, we present a new cascade method for human face detection which exploits several cascades for data not only from image source but also various heterogeneous sensors. It provides more accuracy of real human face detection and reduces a number of classifier to high speed processing in real-time detecting. For this approach, we need to synchronize between each sensor, so that sensors data can be processed at the same time. Using this method, we can improve the accuracy of face detection.

The outline of our paper is as follows: In Section 2, we describe related works for introducing Haar Feature-base Cascade. And time synchronization method for various sensor. Then, in Section 3, we explain a new method using classifiers which process data from various sensors. Section 4 explains implementation of proposed method and shows its experimental results. Lastly Section 5 summarizes the conclusion of our research.

\section{RELATED WORKS}

Haar Feature-based Cascade is fast object detection algorithm [2] using Haar-like features and a cascade of classifiers. It has good detection rate depends on training data. And it calculates 2 frames data per second so that it can process in real-time. This algorithm consists of 4 stages. First stage is haar feature selection. Haar-like features can be made by calculating difference of the sum of pixels of areas inside rectangle. There are many haar features in a frame. This feature has too many operation to service in real-time. For this, it use second stage which has integral method to calculate quickly. And third stage is Adaboost training. Adaboost selects useful haar feature in total haar features using weight of each haar filter [4]. Each selected features can be trained data to classify true positive. Then, Adaboost can compose strong classifier which is consists of weak classifier. Last stage is to make cascading classifiers. These cascading classifiers is step by step method which is made by several weak classifiers. Firstly, top simple classifier judge the features whether it is true or false. If first classifier classify features as a true positive, it could be passed the next stage which consists of another weak classifiers. This method can reduce a lots of operation by using classifier cascading. Once, a classifier reject the features, It is regarded as false so that it cannot be passed next classifiers. All features pass the classifier cascading, it is targeted as an object.

There are various researches for processing sensor data from heterogeneous sensors[5-7]. Those sensors data are detected different elements respectively so that enhance the accuracy of detection result. This various elements can be used source of classifier cascade. For example, temperature sensor and weight sensor are a great help to detect real human.

Previous face detection approaches consider only multimedia source from a camera. Those methods have so many classifiers for high detection rate that it takes a great time. Our research goal is to enhance the true positive rate of detection using various sensors to reduce a number of classifiers. 


\section{FACE DETECTING USING HeTEROGENEOUS SENSORS}

In this section, we present a new architecture of face detection system using heterogeneous sensors for detecting real-human. Unlike typical face detecting systems, our system has additional classifiers to process various sensor data

\subsection{Key features}

This system has several key features as follows:

(1) It offers minimum number of cascades so that it reduce volume of operation. Typically, to enhance the true positive rate of detection, it would be a lot of cascades such as face cascade, eye cascade, nose cascade or mouth cascade. However, there is a trade-off between accuracy of result and processing speed. The more cascade are contained, the more it takes a time. our system select minimum number of cascades for high speed processing

(2) It offers time-stamp for processing the various sensors data at the same time. And those data come separately. Thus, it needs to synchronization for various sensors data. Our system set the time-stamp for synchronization.

(3) It offers real-face detecting except picture, doll using feature of human. A surveillance system has to detect real human, this system can extract feature of human using sensor such as temperature sensor. Moreover, multiple source enable system to detect various sense not only vision but also touch sense, weight sense, heat sense and so on. It is helpful to detect objects exactly which we want. In a cascade step, it judges sensor data whether it is necessary or unnecessary by using several classifier. If it is considered as true positive, it is passed next phase of cascade from other sensors.

\subsection{System Model}

The overall operation of our system model as shown fig 1. Our system model consists of train phase, synchronization phase and cascading phase. This operations shall be explained bellows.

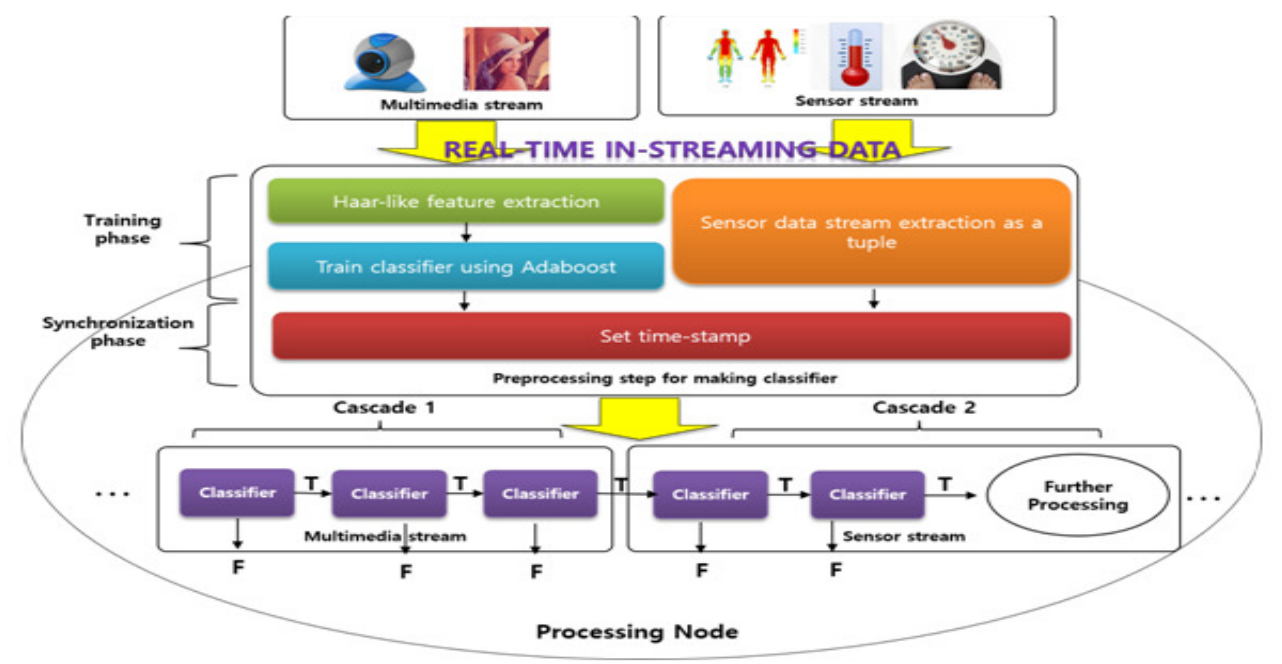

Figure 1. The overall model of face detecting system using heterogeneous sensors 


\subsubsection{Training Phase}

In training phase, there are two section to make classifiers. First section is for training multimedia data from camera sensor in real time. Haar-like feature extraction and Adaboost make the classifier based on multimedia data. Second section is for extraction tuple from various sensors data except camera. Its section collects the sensors data and extracts tuple in real time. However, it is different time between each section yet. So it needs synchronization of time in next phase.

\subsubsection{Synchronization Phase}

In synchronization phase, it synchronizes the time between haar-like features and sensor tuples. Those sensors data are detected different elements respectively. Thus, it has different time stamp. To synchronize their time, sensor data set their time every frame-rate cycle so that it reduces volume of calculation. If its frame rate is $12 \mathrm{fps}$, other sensors data set their time every 12 frame. The time of Multimedia is standard-time. Figure 2 shows an example of this method.

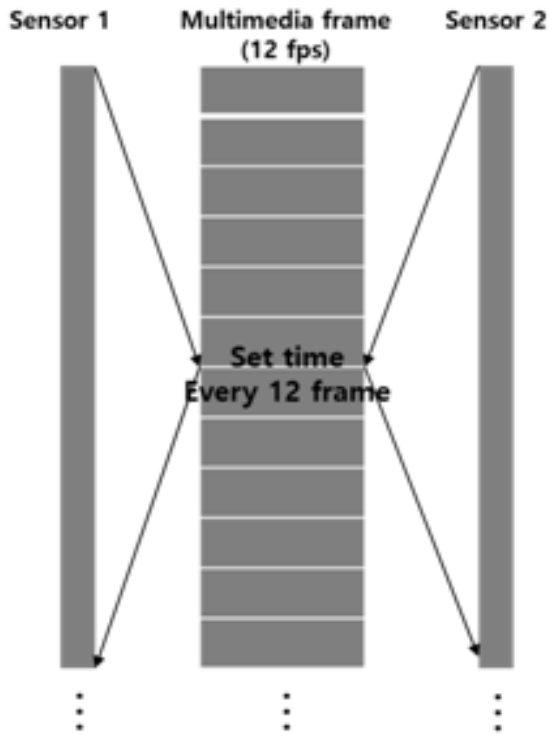

Figure 2. An example of synchronization method

\subsubsection{Cascading Phase}

In cascade phase, it makes robust classifier which consists of week classifier. Its cascades is connected to one another. To detect face, face cascade is set on head stage. Then, other cascades are set on next stage. It is mandatory that prior cascade judges true feature before posterior cascades. If prior cascade judges false, it doesn't pass the opportunity to next cascade. There are cascades made by Adaboost algorithm for multimedia sensor process. The rest of cascades are made by range detector for heterogeneous sensors. The cascade consists of various sensors data classifier as shown figure 3 . 


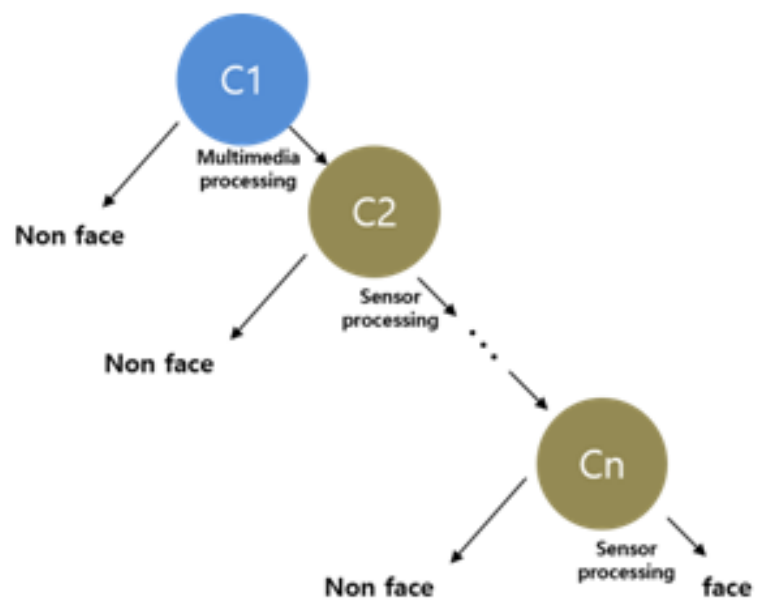

Figure 3. The model of cascade including multiple source processing

\section{IMPLEMENTS}

In this section, we shell show the implementation of our new system. We implement a face detection with a temperature data. There are six cases for this implementation. First is a realhuman detecting with face cascade and no temperature cascade. Second case is the picture of human with face cascade and no temperature cascade. Third case is the picture of human with face cascade and temperature cascade. Fourth case is the real-human with face cascade, eyes cascade and no temperature cascade. Fifth case is a picture of human with face cascade, eyes cascade and no temperature cascade. The last case is a picture of human with face cascade, eyes cascade and temperature cascade. The implement cases of implementation as shown blows.

Table 1. Various cases of implement

\begin{tabular}{|c|c|c|c|}
\hline Case Number & Object Type & The number of Cascades & Additional Sensor \\
\hline Case 1 & Real face & 1 (face) & No sensor \\
\hline Case 2 & Picture & 1 (face) & No sensor \\
\hline Case 3 & Picture & 1 (face) & Temperature sensor \\
\hline Case 4 & Real face & 2 (face, eyes) & No sensor \\
\hline Case 5 & Picture & 2 (face, eyes) & No sensor \\
\hline Case 6 & Picture & 2 (face, eyes) & Temperature sensor \\
\hline
\end{tabular}




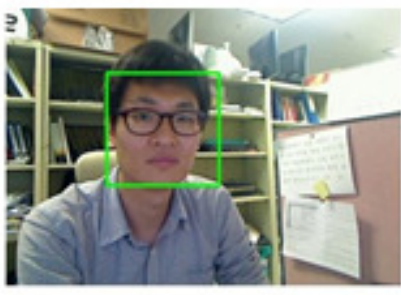

Case 1

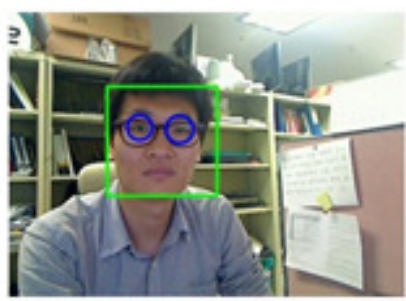

Case 4

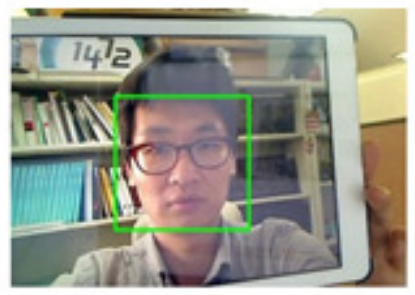

Case 2

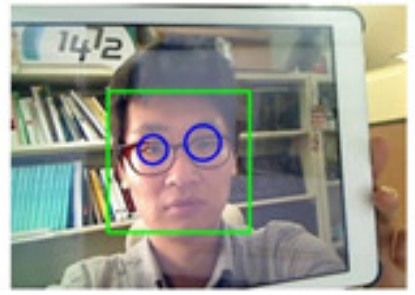

Case 5

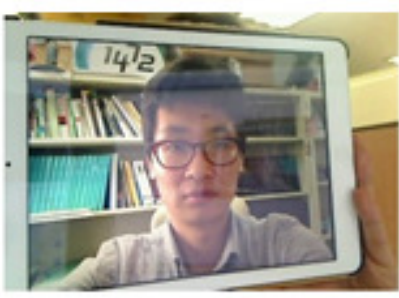

Case 3

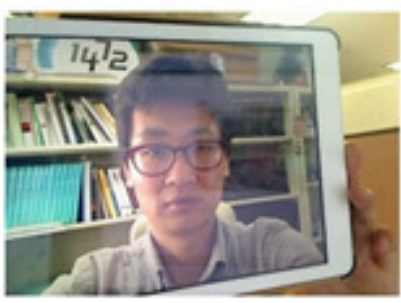

Case 6

Figure 4. Result of implementation

\section{EXPERIMENTAL RESULTS}

We implement this system on 1 node which has Intel ${ }^{\circledR}$ core $^{\mathrm{TM}}$ quad CPU Q6600 $2.40 \mathrm{GHz}$ processors and $8 \mathrm{~GB}$ memory. The experimental results show that real human face-detecting system which has cascades from various sensors enhance the accuracy of detecting real human. Case 2 without temperature cascade detects the face. It is false positive. However, case 3 has no detection of face. It is true negative. Figure 5 shows the relation between the numbers of cascades and elapsed time. Case 4, 5 has eyes cascade additionally. Those case take a lot time to calculate than case $1,2,3$. Because it contains many operation to extract haar feature since it contains eyes cascade. Case 2 is faster than case 3 . It means that the more cascades are contained in that, the more it takes a time to detect. Because cascade based on multimedia data which contains many 'for statement', it can reduce the elapsed time by reducing cascade or using cascade form sensor data instead of multimedia data. Although case 6 contains eyes cascade, it processes the detection step faster than case 4 and 5. Because Temperature cascade does not pass the opportunity to eyes cascade. As a result, the composition consists of various cascades from heterogeneous sensors are the helpful to detect of real human-face.

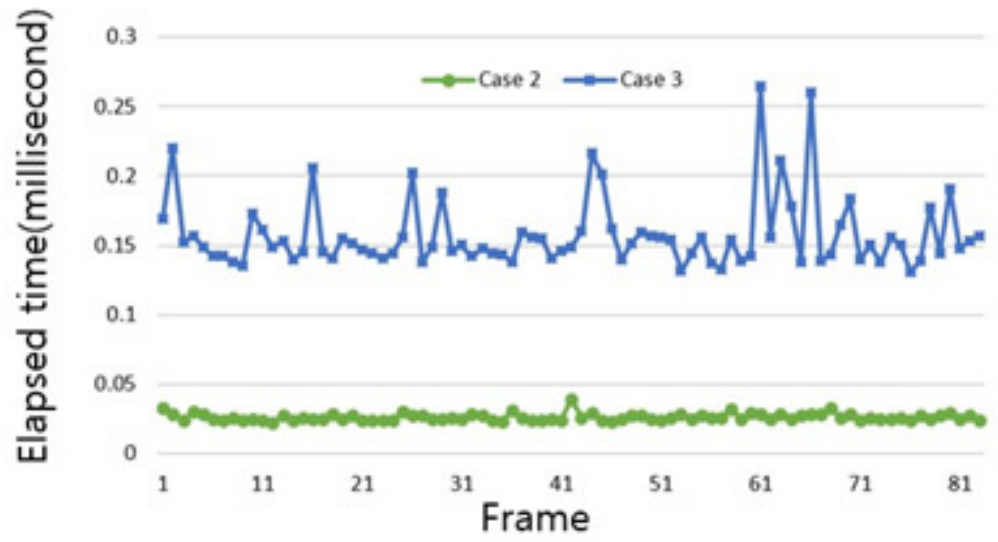




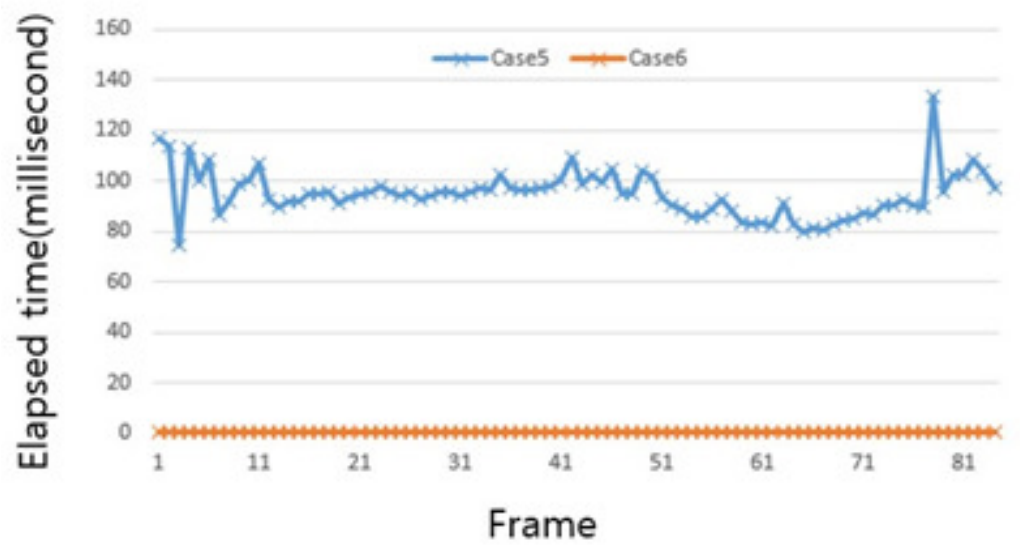

Figure 5. Result of experiments

\section{CONCLUSIONS}

In this paper, we have presented a cascade method for human face detection in surveillance system which exploits several classifiers for data not only from image source but also various heterogeneous sensors. A typical face detection algorithm such as Haar Feature-based Cascade Classifier gives us an easier way to detect the face. However, it consists of several classifiers which contain complicated arithmetic operations so that it takes a great deal of time to achieve a result. And it is hard to recognize real human face in short time, since previous cascade processes only one image source. Our method can enhance an accuracy of face detection using heterogeneous sensors. It uses a cascade which consists of classifiers. Each classifier processes data from not only image source but also various sensors data. It provides more accuracy of real human face detection and reduces the number of classifiers for high speed processing in real-time detecting.

\section{ACKNOWLEDGMENTS}

This research was supported by Korea university and MSIP(Ministry of Science, ICT and Future Planning), Korea, under the ITRC(Information Technology Research Center) support pro-gram (IITP-2015-H8501-15-1004) supervised by the IITP(Institute for Information \& communications Technology Promotion)

\section{REFERENCE}

[1] Yu, Byunggu, Ranjan Sen, and Dong H. Jeong. "An integrated framework for managing sensor data uncertainty using cloud computing." Information Systems 38.8 (2013): 1252-1268.

[2] P. Viola and M. J. Jones, "Rapid Object Detection using a Boosted Cascade of Simple Features," In Proc. of CVPR 2001.

[3] Gama, João, and Pavel Brazdil. "Cascade generalization." Machine Learning41.3 (2000): 315-343.

[4] VIOLA, Paul; JONES, Michael. Fast and robust classification using asymmetric adaboost and a detector cascade. Advances in Neural Information Processing System, 2001, 14. 
[5] Yu, Byunggu, et al. "On managing very large sensor-network data using bigtable." Cluster, Cloud and Grid Computing (CCGrid), 2012 12th IEEE/ACM International Symposium on. IEEE, 2012.

[6] Jung, I. Y., Kim, K. H., Han, B. J., \& Jeong, C. S. (2014). Hadoop-Based Distributed Sensor Node Management System. International Journal of Distributed Sensor Networks, 2014.

[7] Kui, X., Sheng, Y., Du, H., \& Liang, J. (2013). Constructing a CDS-based network backbone for data collection in wireless sensor networks. International Journal of Distributed Sensor Networks, 2013.

\section{AUTHORS}

Yoon-Ki Kim is currently working toward the ph.D degree in Electronic and Computer Engineering at the Korea University. His research interests include real-time distributed and parallel data processing, IoT, Sensor processing and computer vision.

Du-Hyun Hwang is currently working towards a master's degree at Department of Electrical Engineering, Korea University. His current research interests are distributed parallel computing, computer vision and GPU processing

Chang-Sung Jeong is a professor at the department of EE/CE at Korea University. He received his MS.(1985) and Ph.D.(1987) from Northwestern University, and B.S.(1981) from Seoul National University. Before joining Korea University, he was a professor at POSTECH during 1982-1992. He also worked as an associate researcher at UCSC during 1998-1999.
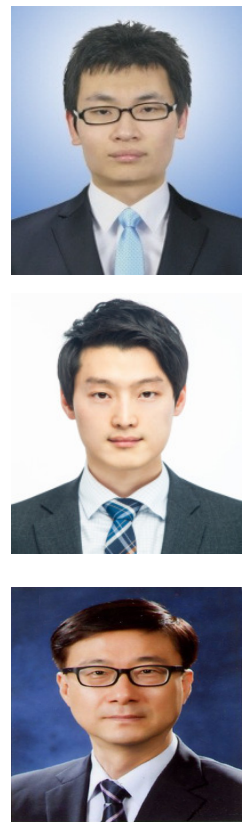